\title{
El peso de lo que no se mide. Principales factores subyacentes en el Índice de Desigualdad de Género del PNUD
}

\section{The impact of what is not measured. Principal underlying factors in the UNDP Gender Inequality Index}

\author{
Dra. AmÉrica Ivonne Zamora Torres ${ }^{*}$ \\ ORCID ID: 0000-0003-1811-4711 \\ DR. RENÉ Augusto MaRÍn LEYvA* \\ ORCID ID: 0000-0002-4782-3798 \\ MTRA. YesiCA DíAz BARAJAS ${ }^{* * *}$ \\ ORCID ID: 0000-0003-4503-8881
}

Universidad Michoacana de San Nicolás de Hidalgo

Recibido: $12 / 10 / 2021$

Aceptado: 10/12/2021

doi: https://doi.org/10.20318/femeris.2022.6620

Resumen. En la actualidad siguen existiendo importantes desigualdades en las condiciones en las que viven y se desenvuelven los hombres y las mujeres en la mayoría de los países del mundo, que en su conjunto constituyen importantes brechas de género en diversos ámbitos, las cuales impiden a las naciones beneficiarse del máximo potencial de ambos géneros en sus procesos de desarrollo. El objetivo de este trabajo es identificar a través de la generación de evidencia empírica, los factores que impactan en la desigualdad de género de 161 países para el año 2020, integrando dimensiones a la propuesta del Índice de Desigualdad de Género (IDG) desarrollada por el Programa de las Naciones unidas para el Desarrollo (PNUD), lo cual permitiría abordar la problemática de una manera más integral. Para realizar este trabajo se utilizó un método de análisis factorial de tipo comprobatorio, considerando las variables que actualmente integran las tres dimensiones del IDG 2020, encontrándose -en concordancia con la teoría analizada- el rol de género femenino como primer factor subyacente, que enmarca a las mujeres como principales responsables del ámbito reproductivo y del hogar, actuando como una primera forma de exclusión que les impide incorporarse en la esfera social, productiva y política con las mismas condiciones de libertades y oportunidades que los hombres.

Palabras clave: mujeres, desigualdad de género, desarrollo humano, Índice de Desigualdad de Género, análisis factorial.

Abstract. Currently, there are still significant inequalities in the conditions in which men and women live and act in most of the countries of the world, which as a whole, constitute important gender gaps in various areas, that do not allow countries to benefit with the maximum potential of both genders in their development processes, also preventing women from reaching their maximum human potential. The objective of this paper is to identify, through the generation of empirical evidence, the factors that impact gender inequality in 161 countries

\footnotetext{
*america.zamora@umich.mx

*rene.marin@umich.mx

*** yesidb_09@hotmail.com
} 
by the year 2020, integrating dimensions to the proposal of the Gender Inequality Index (GII) developed by the United Nations Development Program (UNDP), which would allow addressing the problem in a more comprehensive way. To achieve this objective, it was used a verifiable factor analysis method, considering the variables that currently make up the three dimensions of the GII in 2020, finding -according with the theory analyzed- the role of the female gender as first underlying factor, which frames women as main responsible for the home and reproductive sphere, acting as a first form of exclusion that prevents them from joining the social, productive and political spheres with the same conditions of freedom and opportunities that men.

Keywords: women, gender inequality, development, Gender Inequality Index, factor analysis.

\section{Introducción}

La igualdad entre mujeres y hombres en todos los ámbitos es reconocida a nivel nacional e internacional como una condición necesaria para alcanzar sociedades más justas, equitativas e inclusivas, por lo que desde los más altos documentos normativos se han plasmado objetivos para fomentarla. Como lo ha señalado la Organización de Naciones Unidas (ONU, 2015), La incorporación sistemática de una perspectiva de género en la implementación de la Agenda 2030 para el Desarrollo Sostenible es muy importante, ya que no es posible realizar todo el potencial humano y alcanzar el desarrollo sostenible si se sigue negando a la mitad de la humanidad el pleno disfrute de sus derechos humanos y sus oportunidades, por lo que se hace imprescindible garantizar que las mujeres tengan acceso a una educación de calidad, recursos económicos y participación política, así como a un trabajo decente, atención de la salud y una vida sin violencia ni discriminación. Como lo ha mencionado la Comisión Económica para América Latina (CEPAL, 2018) la igualdad entre los géneros no es solo un derecho humano fundamental, sino la base necesaria para conseguir un mundo pacífico, próspero y sostenible, que beneficie a las sociedades y a la humanidad en su conjunto.

Si bien se reconoce que se han producido importantes avances a nivel mundial con relación a la igualdad entre los géneros a través de los acuerdos internacionales y las políticas implementadas en los últimos años, las mujeres siguen sufriendo discriminación y violencia en todos los lugares del mundo. En las agendas de gobierno se ha reconocido explícitamente la persistencia de condiciones que obstaculizan el desarrollo de las mujeres, al limitarlas, segregarlas, discriminarlas o excluirlas en diversos ámbitos, por lo cual es fundamental diseñar políticas públicas de corto, mediano y largo plazo que coadyuven a combatir las causas históricas y estructurales que impiden a las mujeres alcanzar su máximo potencial humano, para que puedan participar activamente en los procesos de desarrollo con equidad (Diario Oficial de la Federación, 2013).

En este sentido, el adecuado diseño de políticas públicas debe contemplar la desagregación y atención de las principales causas que subyacen al problema público (Merino, 2013), por lo cual, contar con información suficiente e indicadores adecuados que permitan dimensionar la situación problemática, constituye un gran aporte para el análisis de la política pública. El IDG del PNUD ha sido comúnmente utilizado en los últimos años como un referente internacional para cuantificar la desigualdad de género y analizar su evolución, mostrando los logros no realizados debido a las diferencias en las condiciones entre hombres y mujeres, en las dimensiones de salud reproductiva, empoderamiento y merca- 
do laboral, con valores que van de 0 -igualdad perfecta- a 1 -desigualdad total- (PNUD, 2010). Sin embargo, también se ha reconocido que las principales herramientas y prácticas actuales para dimensionar la desigualdad de género, no son suficientes para brindar la información necesaria que respalde el debate público y la correspondiente toma de decisiones que abonen a su eliminación (PNUD, 2019).

Este trabajo tiene como objetivo identificar, a través de la generación de evidencia empírica, los factores que impactan en la desigualdad de género de 161 países para el año 2020, integrando dimensiones a la propuesta del IDG del PNUD, que representan importantes factores estructurales que subyacen a la desigualdad de género, ya que el IDG considera para su cálculo actual elementos comúnmente cuantificados por la mayoría de las naciones, dado su impacto en otros ámbitos como el económico y el político, pero deja de lado otros aspectos tradicionalmente considerados del ámbito privado, como lo son la inequitativa participación en el cuidado y las responsabilidades del hogar y la formación del suficiente capital humano de las mujeres, careciendo generalmente ambos factores de datos sistemática y permanentemente contabilizados a nivel internacional, dificultando el análisis de su impacto y evolución, así como su comparación entre regiones y países.

La estructura de este trabajo contempla en su primer apartado el contexto de la desigualdad de género y sus implicaciones en el desarrollo humano; en el segundo se abordan de forma teórica y referencial diferentes factores que impactan en la desigualdad entre hombres y mujeres, partiendo de las tres dimensiones consideradas en el IDG; en el tercer apartado se explica la metodología del análisis factorial; para dar paso a un cuarto apartado, donde se presentan los resultados correspondientes a los factores subyacentes del IDG en 2020 y finalmente, se presentan las conclusiones del estudio.

\section{La desigualdad de género y el desarrollo humano}

La igualdad de género puede entenderse como una condición ideal en que las personas disfrutan de iguales derechos políticos, económicos, sociales y educativos (entre otros) basado en estatutos jurídicos y en el principio de no discriminación por la diferencia sexual, que resulta en que mujeres y hombres tengan las mismas oportunidades de vida (Zamudio et al., 2014). La búsqueda de la igualdad de género implica transitar por medidas de equidad como un principio de justicia encaminado a acelerar la igualdad de facto, que implican algunas veces beneficios, ventajas, cuotas y en general acciones que pongan a hombres y mujeres en un mismo plano, las cuales no constituyen acciones discriminatorias, sino medidas positivas de carácter temporal que posibiliten a hombres y mujeres gozar de las mismas libertades y oportunidades (Magaña, 2018).

Las inequidades y desigualdades de género representan un problema multifactorial que ha sido promovido y legitimado explícita y simbólicamente por la cultura, llegando a permear en todas las esferas de la vida humana, por lo que prácticamente en casi todas las actividades humanas -sociales, culturales, religiosas, políticas, personales, etc.-, que impliquen relaciones entre hombres y mujeres, se generan inequidades relacionadas con 
el género que derivan en situaciones de desigualdad en las posibilidades y oportunidades de vida, que ponen en desventaja principalmente a las mujeres (Zamudio et al., 2014).

Retomando las ideas de Sen (2000), el desarrollo humano rebasa el ámbito económico y debe concebirse como un proceso de expansión de las libertades reales de que disfrutan los individuos para lograr resultados valorados, por lo que exige la eliminación de las principales fuentes de privación de la libertad, entre las que se encuentran las privaciones sociales sistémicas, que afligen tal vez a la mayoría de la población. Se ha observado que la desigualdad de género se relaciona directamente con una pérdida de desarrollo humano, por lo que los países que han logrado reducir las desigualdades en dicho ámbito, han reducido primeramente las principales desigualdades de género (PNUD, 2019).

Las desigualdades en las que viven las mujeres y los hombres han generado brechas de género en diversas dimensiones, ocasionando que un gran número de mujeres viva en condiciones que no tendrían si pudieran evitarlo, impidiendo así mismo, que un gran porcentaje de la población alcance su máximo potencial humano y disfrute de todas las libertades que el grado de desarrollo pudiera brindarle. Como lo menciona Daeren (2001), un modelo de desarrollo es equitativo y eficiente, en la medida que pueda satisfacer las diversas necesidades de las personas en condiciones de igualdad de oportunidades y de equidad de trato, por lo que es imprescindible que en las políticas se aplique un enfoque de género que permita maximizar los aportes de hombres y mujeres al desarrollo.

Como una herramienta para cuantificar el nivel de desarrollo humano y evidenciar sus desigualdades, desde 1990 el Programa de las Naciones Unidas para el Desarrollo ha publicado el Índice de Desarrollo Humano (IDH), como un índice compuesto que mide el promedio de los avances de las personas en tres dimensiones básicas: una vida larga y saludable, un nivel de conocimientos y un nivel de vida digno (PNUD, 2019).

Posteriormente, respondiendo a la reconocida importancia de cuantificar el avance hacia un desarrollo humano más igualitario, el PNUD ha incorporado desde 2010 el cálculo del IDG dentro de sus informes de Desarrollo Humano, como un índice compuesto que muestra la pérdida de desarrollo humano resultado de la desigualdad entre mujeres y hombres, midiendo la diferencia en el logro entre ambos sexos en tres dimensiones: salud reproductiva, empoderamiento y mercado laboral. Cuando el IDG tiene un valor cercano a cero indica que el desarrollo es igualitario, mientras que cuando se acerca a uno muestra la existencia de una amplia desventaja para las mujeres con relación a los hombres. Se reconoce, sin embargo, que existen otros factores que influyen también en el bienestar de las mujeres, como son el uso del tiempo, acceso a activos, violencia doméstica y empoderamiento, los cuales no han sido incluidos en el cálculo del IDG por falta de información confiable y oportuna al respecto (PNUD, 2010).

Algunas de las principales consideraciones sobre la desigualdad de género que se presentan en el informe del PNUD (2019) son las siguientes:

- No existe plena igualdad de género en ningún país del mundo.

- Los avances para un desarrollo humano más igualitario se han ido ralentizando en los últimos años, derivando en amplias brechas de género. 
- Las desigualdades de género rebasan la distribución desigual del progreso derivado del desarrollo económico.

- Las problemáticas en la desigualdad siguen dinámicas muy diferentes, dado que se presentan dentro de los hogares, entre personas, entre grupos, entre países y dentro de los países.

- Persisten normas sociales arraigadas culturalmente para preservar las estructuras de poder tradicionales.

- Las mujeres en la actualidad son las más cualificadas de la historia, pero sus méritos aún no se reflejan exitosamente en el trabajo remunerado, dado el peso de su rol reproductivo.

- Se observa en general una paridad en la participación política básica, en tanto que, a mayor concentración del poder, las mujeres quedan subrepresentadas.

- Los datos de género subestiman los roles y contribución de las mujeres y presentan problemas de cantidad y calidad para reflejar la realidad.

Estas afirmaciones dejan en evidencia la existencia de factores que subyacen a la desigualdad de género adicionales a los que contempla el IDG, que resultan más difíciles de abordar, ya que preservan una estructura patriarcal arraigada culturalmente, haciendo comprensible la ralentización del proceso de igualdad en los últimos años. El problema de la insuficiencia de cantidad y calidad de los datos a nivel global sobre los roles de género, se considera especialmente relevante, ya que impide incorporar a la medición sistematizada de la desigualdad de género, importantes factores estructurales que fomentan la exclusión de las mujeres de la esfera pública y perpetúan las desigualdades en diferentes ámbitos.

\section{Acercamiento teórico y referencial de la desigualdad de género}

Los roles de género tienen implicaciones en todos los ámbitos de actuación de hombres y mujeres, como parte sustancial de las sociedades, por lo cual, su estudio se puede abordar desde diversos enfoques y perspectivas teóricas, dependiendo del alcance y el objeto de estudio que se pretenda alcanzar. Sin embargo, este trabajo acota su análisis, en concordancia con el objetivo de investigación presentado, a las tres dimensiones que se consideran en el cálculo del IDG: la salud reproductiva, el empoderamiento y el trabajo, por lo que a continuación, se presenta su aproximación teórica y referencial.

\subsection{El género y sus roles}

De acuerdo con Lamas (1986) desde la antropología, el género refiere a un orden simbólico con el que cada cultura elabora la diferencia sexual e instaura los papeles sexuales con base en el ámbito biológico, marcando las diferencias en la participación de hombres y mujeres en las instituciones sociales, económicas, políticas y religiosas, incluyendo 
valores, actitudes y expectativas de una sociedad dada, que conforman el pilar de las relaciones sociales basadas en las diferencias biológicas y constituyen una forma primaria de las relaciones de poder.

Por su parte, Aguilar et al. (2013, citand o a Arellano, 2003), distinguen ente los conceptos de sexo y género, señalando que el sexo corresponde a una categoría biológica, mientras que el concepto de género hace referencia a la construcción social que resulta del hecho de ser hombre o mujer, que define expectativas y valores, así como la interrelación entre hombres y mujeres y sus relaciones de poder y subordinación en una sociedad determinada. Mencionan (2013, citando a Maya, 2003) que en el contexto latinoamericano, los hombres son los que toman las decisiones importantes acerca de los gastos familiares y tienen mayor libertad de expresión y de elegir su estilo de vida, mientras que para las mujeres, la maternidad y el cuidado de hijos, esposo, enfermos y ancianos, sigue siendo una exigencia social, aunque estas responsabilidades se han venido articulando cada vez más con el desempeño y la realización profesional, especialmente en los contextos más industrializados.

Asimismo, Martínez (2015, citando a Medina y Márquez, 2010) ha señalado que, desde la época de la Ilustración, ha existido una dicotomía entre lo público y lo privado, situando a mujeres y hombres en una división sexual del trabajo caracterizada por una disparidad en jerarquías y relaciones de poder de género. Señala también (2015, citando a González, 2009), que en diversas sociedades aún existe una cultura patriarcal perpetuada generacionalmente, basada en roles de género diferenciados y complementarios, que reducen a las mujeres a las labores domésticas y del cuidado de las personas, creando relaciones de poder asimétricas que dejan a las mujeres en una condición subordinada y de desventaja respecto de los hombres.

\subsection{Salud reproductiva}

Ramos (2006), menciona que el concepto de salud reproductiva rebasa la ausencia de enfermedades, refiriendo a un estado de bienestar físico, mental y social de la persona en todos los aspectos relacionados con el sistema reproductivo, sus funciones y procesos. Citando a Cook, et al. (s.f.), Ramos (2006) pone en evidencia que la imposibilidad de regular y controlar la propia fecundidad, constituye una violación a los derechos humanos que afecta la salud y el bienestar de las personas directamente involucradas, especialmente de las mujeres.

La CEPAL (2004) ha señalado que la autonomía del cuerpo de las mujeres representa sus posibilidades para acudir a recibir atención especializada en salud sexual y reproductiva que, a su vez, repercute en una menor mortalidad materna y menores embarazos no deseados, además de habilitarlas para contribuir a la salud familiar, siendo la mortalidad materna un indicador ampliamente utilizado para evidenciar las inequidades sociales. Citando a Gómez (1997), menciona que las principales causas de la muerte materna pueden prevenirse y se relacionan generalmente con hemorragias graves, eclampsia y abortos realizados en condiciones inseguras. 
Gamboa y Valdés (2015: 11) retoman el concepto de la Organización Mundial de la Salud (OMS) para definir la mortalidad materna como "la defunción de la mujer durante el embarazo, parto o dentro de los 42 días siguientes a la terminación del embarazo, debida a cualquier causa relacionada con o agravada por el embarazo o su atención..." Menciona también (2015, citando a IPAS, 2015) que la muerte materna es el reflejo de una suma de inequidades y discriminaciones que mantienen a las mujeres alejadas de la educación y la toma de decisiones, reflejándose en la poca o nula importancia que brindan al cuidado de su salud, coartando sus opciones reproductivas y afectando severamente su salud hasta llegar a la pérdida de la vida.

Por otra parte, el Fondo de Población de las Naciones Unidas (UNFPA) ha señalado que el embarazo adolescente involucra costos de oportunidad para el desarrollo social y el crecimiento económico del país, dado que las adolescentes embarazadas por lo general interrumpen su educación y difícilmente la retoman, lo cual dificulta su inserción en el mercado laboral y sus oportunidades para obtener un trabajo estable y decente (UNFPA, 2020). Se ha observado que el embarazo adolescente aumenta por falta de acciones preventivas, educativas y de acceso a servicios de salud, presentando las adolescentes un mayor riesgo de complicaciones durante el embarazo, parto o puerperio, aunado a que la mayoría de dichos embarazos son no deseados, por lo que el aborto les representa una posible alternativa, siendo este procedimiento la quinta causa de muerte materna (Diario Oficial de la Federación, 2013).

\subsection{Empoderamiento femenino}

En palabras de Patil y Banasode (2018), el empoderamiento es un proceso social multi-dimensional que ayuda a las personas a obtener control sobre sus propias vidas, fomentando el poder (entendido como la capacidad de implementar) en las personas, que les permite actuar en temas que consideran relevantes de sus propias vidas, sus comunidades y sus sociedades.

Para lograr el empoderamiento femenino, es necesario que las mujeres puedan trascender del ámbito doméstico, así como contar con mayores capacidades y habilidades para interactuar efectivamente en el ámbito público e influir en las cuestiones importantes de la comunidad, ante lo cual, se deben implementar mayores estrategias de política pública que contemplen la participación de las mujeres en todas las etapas del proceso, que permitan incorporar sus necesidades y puntos de vista para la resolución de los problemas públicos (Ikanowicz y Barbara, 2016).

Como parte del empoderamiento, se ha señalado que la participación política de las mujeres es una exigencia para avanzar hacia una cultura democrática efectiva, que contribuye al buen gobierno y al logro de un desarrollo sostenible, observándose que el promover que las mujeres ocupen altas responsabilidades en política tiene un efecto multiplicador para empoderar a más mujeres en todas las esferas de sus vidas, generando nuevos roles y prototipos de mujeres, distintos de los tradicionales, que permiten 
erradicar prejuicios discriminatorios y contribuyen a la sensibilización de la sociedad en el tema del género, favoreciendo la incorporación de diversas medidas positivas que hacen a la esfera pública más accesibles para el género femenino. Sin embargo, se ha reconocido también, que la participación política de las mujeres sigue siendo limitada por diversos factores estructurales, entre los que se encuentran actitudes culturales basadas en modelos patriarcales, estereotipos sexistas y roles tradicionales de hombres y mujeres (ONU, 2014).

Entendiéndose el poder como el acceso a recursos físicos e ideológicos en una relación social, se ha señalado que la distribución desigual del poder y los obstáculos para que las mujeres participen activamente en los procesos de decisión en el espacio público, impiden el pleno ejercicio de sus derechos y su ciudadanía, por lo cual, el empoderamiento femenino debe considerarse también como un concepto político que rebasa la participación política formal, haciéndose necesario detonar cambios efectivos en las relaciones de poder entre ambos géneros (CEPAL, 2004).

Adicionalmente, la educación representa otro elemento esencial para el empoderamiento que, además de ser un derecho vinculado al desarrollo pleno de las personas, incide decisivamente en las oportunidades y la calidad de vida de los individuos, las familias y las colectividades, cumpliendo un papel clave en la distribución de las oportunidades de bienestar, en particular en sociedades como la latinoamericana, caracterizada por grandes desigualdades sociales. La educación es un factor fundamental del desarrollo, por lo cual, deben procurarse mayores niveles de acceso, conclusión de los ciclos escolares y mayores logros cualitativos en materia de aprendizaje, como una condición necesaria para garantizar los derechos sociales básicos, influyendo también positivamente en el crecimiento económico y la equidad, mediante la acumulación de capital humano (CEPAL, 2005).

En este mismo sentido, se ha señalado que la educación es la variable determinante del éxito en el mercado laboral, ya que constituye el capital humano con el cual los individuos pueden incorporarse con mejores conocimientos y habilidades dentro del mercado laboral, traduciéndose en mayor productividad e ingresos, existiendo evidencia de que las personas con mayores estudios tienen mayores ingresos en comparación con personas de menores estudios (Angulo et. al, 2012).

Por su parte, Aguayo y Lamelas (2011, citando a Saraví (1997) y Janssen (2005), señalan que aunque la educación es el factor reconocido de mayor incidencia en la participación laboral, que contribuye a alcanzar mejores posiciones y niveles de ingreso, la educación explica sólo una parte de las diferencias de ingresos y oportunidades entre ambos géneros, existiendo una segmentación laboral con un mercado primario más estable y mejor remunerado para los hombres y uno secundario más inestable y peor pagado para las mujeres.

\subsection{La participación en el trabajo}

Noguera (2002) aborda el trabajo desde un concepto amplio, que no necesariamente consiste en una actividad pura y exclusivamente instrumental, sino que puede tener 
recompensas intrínsecas a la misma, e incluso puede tener en ella misma su propio fin. Menciona a Marx (s.f.), quien lo concibe desde tres dimensiones: el trabajo es una actividad orientada a un fin, pero también es interacción social y comunicación, así como autoexpresión práctica del ser humano, que desarrolla en él un libre juego de fuerzas vitales físicas y espirituales; por lo cual, es importante valorar no solo el trabajo remunerado, sino también el no remunerado que genera un alto valor social.

De acuerdo con lo señalado por la ONU Mujeres (2015) el trabajo remunerado femenino tiene un efecto positivo en el desarrollo de las naciones, por lo que, cuando el número de mujeres ocupadas aumenta, las economías crecen. Indica también que según estudios efectuados en varios países, el aumento de la participación de las mujeres en la fuerza de trabajo, así como una reducción de la disparidad entre la participación de ambos géneros en la fuerza laboral, produce un crecimiento económico más acelerado, al igual que, al incrementar la proporción de los ingresos del hogar controlados por mujeres, procedentes de lo que ganan ellas mismas o de transferencias de dinero, se modifican los patrones de gasto en formas que benefician directamente a sus hijas e hijos.

Por otro lado, como lo ha señalado la CEPAL (2004, citando a Espino, 2003), la posición que ocupan las mujeres como principales responsables del ámbito doméstico y del cuidado de las personas, opera como su primera forma de exclusión de los ámbitos económico, político y social, limitando su correcta incorporación en el mercado laboral y en su participación en la esfera pública, mermando asimismo su pleno desarrollo humano. En este sentido, se ha reconocido que las iniciativas que buscan fomentar la redistribución del tiempo entre los géneros para compatibilizar las responsabilidades del ámbito privado con los avances de la esfera pública, siguen siendo insuficientes, por lo que los hombres generalmente no participan en la misma proporción que las mujeres en el trabajo no remunerado que demanda la vida familiar y social (CEPAL, 2004).

\section{Metodología}

Para llevar a cabo este estudio, se realizó un análisis factorial por correspondencias, dado que, como señalan López-Roldán y Fachelli (2016), en cualquier fenómeno social en el cual se hayan identificado un conjunto de variables manifiestas, existirá también una serie de relaciones latentes o causas no manifiestas de su relación y para encontrar dichas relaciones, el análisis factorial proporciona un número reducido de factores o de dimensiones subyacentes al conjunto de variables originales, que conjuntamente afectan el mismo fenómeno. Es por ello que la interpretación que se hace de los nuevos factores, sólo es posible en el contexto de una problemática previamente establecida que justifique la pertinencia de las variables analizadas y de sus posibles relaciones en el marco de una teoría o un modelo más o menos elaborado.

El análisis factorial permite explicar una estructura subyacente que no puede ser observada a primera instancia en un conjunto de variables observables, al encontrar un número reducido de factores subyacentes comunes (K factores) que linealmente recons- 
truyen las p variables originales (Guillermo y García, 2010). El Análisis Factorial de Correspondencias, fusiona el Análisis de Proximidades con el Análisis de Componentes Principales, logrando un análisis de similaridad (proximidad) cuyos principales objetivos son los siguientes (Callealta, 2005):

- Descubrir las relaciones de atracción-repulsión entre las variables.

- Descubrir las relaciones de proximidad existentes entre las distintas modalidades de una misma variable cualitativa.

- Visualizar y caracterizar de forma simple las relaciones anteriores en un espacio de dimensión lo más reducido posible.

El análisis de la similaridad que existe entre las modalidades de un atributo -variables representadas por sus respectivas distribuciones de frecuencias-, condicionadas a su vez por la distribución de modalidades de otro atributo, nos permite analizar la homogeneidad de éstos en dos espacios diferentes, uno de dimensión q y otro de dimensión p; para lo que se introduce y emplea la distancia de Benzecri, que es una ponderación entre los puntos de manera inversamente proporcional a sus frecuencias (Cox y Cox,1994).

Para realizar la operacionalización del modelo en este trabajo, se utiliza el método de extracción de factores o estimación de cargas factoriales, mediante el programa estadístico Statistical Package for Social Sciences (SPSS), de tal forma que se pueda determinar la dependencia y la asociación entre las variables que componen el IDG a partir de la revisión de la intensidad de las atracciones y repulsiones de sus componentes, que permite explicar la varianza total del fenómeno analizado y no solamente la varianza común de la matriz de las variables originales.

Para verificar la pertinencia de la aplicación del análisis factorial, se aplica el test de Kaiser, Meyer y Olkin (KMO) y la prueba de esfericidad de Bartlett, que permiten confirman la validez y la significancia del modelo, siendo que la medida KMO evalúa la relación entre los coeficientes de correlación de la matriz, mostrando valores que van entre 0 y 1 , de tal forma que entre más cercano se encuentre su valor a 1, muestra mayor correlación entre las variables; por su parte, la prueba de esfericidad de Bartlett determina la pertinencia del modelo, mostrando su validez cuanto su valor es menor al nivel de significancia, con lo cual se rechaza la hipótesis nula de esfericidad que afirma que la matriz de correlaciones es igual a una matriz de identidad y se confirma que existen correlaciones significativas entre las variables (Zamora y Thalheim, 2020).

Los resultados del análisis factorial se interpretan por diferentes matrices, siendo la matriz de correlaciones, la que permite identificar la fuerza de las correlaciones entre cada par de las variables analizadas, mediante coeficientes que toman valores entre $1 \mathrm{y}$ menos 1, mostrando una correlación más fuerte entre mas cercano se encuentre su valor de 1, indicando el signo positivo una relación directa y el signo negativo una relación inversa; la matriz muestra al pie una nota con el valor del determinante, que entre más cercano se encuentre de 0 , indica que las variables del modelo se encuentran linealmente relacionadas, por lo que el análisis factorial es pertinente (Zamora y Thalheim, 2020). 
Posteriormente, en la matriz de extracción de factores, se muestran los autovalores de los componentes, que permiten identificar la cantidad de la varianza total explicada por cada uno, extrayéndose aquellos que muestren autovalores superiores a 1, para finalmente, mostrarlos en la matriz de componentes principales, que constituye la solución factorial, en donde se muestran las correlaciones de cada uno de los componentes extraídos con cada una de las variables originales.

Dada la gran cantidad de factores determinantes de la desigualdad de género identificados en la teoría, las variables que se incluyen al modelo de análisis factorial, corresponden a los componentes femeninos que integran el cálculo del IDG del PNUD, que contempla tres dimensiones integradas por cinco variables (PNUD, 2014):

La primera dimensión corresponde a la salud reproductiva, que se conforma por dos variables, la tasa de mortalidad materna, que refiere al número de mujeres que mueren en parto o puerperio por cada 100,000 nacidos vivos, y la tasa de natalidad en adolescentes, que refiere a los nacimientos por cada 1,000 mujeres de 15 a 19 años de edad. Estos datos se obtuvieron del IDG publicado en el Reporte de Desarrollo Humano 2020 (PNUD, 2021).

La segunda dimensión aborda el empoderamiento, que se integra por dos variables, la educación, medida por el porcentaje de mujeres de 25 años y más que cuentan al menos educación secundaria completa, y la representación política, tomada por el porcentaje de mujeres que ocupan escaños parlamentarios, ambos con datos obtenidos del IDG publicado en el Reporte de Desarrollo Humano 2020 (PNUD, 2021).

Finalmente, la tercera dimensión que alude al mercado laboral, se mide con la variable de la participación en el trabajo, que indica el porcentaje de mujeres de 15 años y más que participan en el mercado laboral, con datos obtenidos del IDG publicado en el Reporte de Desarrollo Humano 2020 (PNUD, 2021).

El modelo de análisis factorial considera la información del Índice de Desigualdad de Género publicado en el Reporte de Desarrollo Humano 2020 (PNUD, 2021), que muestra las 5 variables que integran el índice para189 países, con la información más recientes de cada uno de ellos para dicho año, de los cuales, 66 países pertenecen al grupo de desarrollo humano muy alto, 53 al de desarrollo humano alto, 37 al de desarrollo humano medio y 33 al grupo de desarrollo humano bajo. Sin embargo, dado que en el mencionado IDG 2020 no todos los países presentan datos para todos sus componentes y que el modelo no procesa campos sin valores, para su operacionalización se consideraron únicamente los 161 países que muestran datos para cada una de las variables.

\section{Resultados}

Para presentar los resultados del trabajo, se muestran primeramente los principales estadísticos descriptivos del modelo, seguidos de la matriz de correlaciones de las variables, los resultados de la prueba de KMO y Bartlett, la extracción de componentes y finalmente, los factores subyacentes resultantes en la matriz de componentes. 


\subsection{Estadísticos descriptivos}

En la siguiente tabla (tabla 1) que contiene los estadísticos descriptivos de los componentes del IDG, se puede observar que la media del IDG en 2020 para los 161 países analizados es de 0.341 , lo cual representa la pérdida de desarrollo humano de las mujeres respecto de los hombres en las tres dimensiones mencionadas, siendo Suiza el país con menor desigualdad de género, con un IDG cercano a 0 (0.025), seguida de Dinamarca (0.038), mientras la mayor desigualdad de género se presenta en Yemen (0.795), seguida de Chad (0.710).

Tabla 1. Estadísticos descriptivos.

\begin{tabular}{|l|c|c|c|}
\hline \multicolumn{4}{|c|}{ Estadísticos descriptivos } \\
\hline & Media & Desviación estándar & № de análisis \\
\hline Índice Desigualdad de Género & .34130 & .188591 & 161 \\
\hline Tasa Mortalidad Materna & 141.09 & 211.484 & 161 \\
\hline Tasa natalidad en adolescentes & 46.701 & 40.2594 & 161 \\
\hline Mujeres en escaños parlamentarios & 24.007 & 11.5380 & 161 \\
\hline Mujeres con secundaria & 61.590 & 29.1289 & 161 \\
\hline Mujeres en fuerza laboral & 51.857 & 15.3694 & 161 \\
\hline
\end{tabular}

Fuente: Elaboración propia en SPSS, con datos del PNUD, 2021.

Analizando los componentes del IDG del reporte 2020, en la dimensión de la salud reproductiva se puede ver que, en estos países, anualmente mueren en promedio 141 madres por cada 100,000 nacimientos, siendo los países con las menores tasas de muerte materna Noruega, Italia, Polonia y Belarús, con un valor de 2 respectivamente; en contraparte, los países con las mayores tasas son Chad $(1,140)$, seguido por Sierra Leona $(1,120)$. Respecto de la natalidad en adolescentes, se observa que en los países analizados, se tienen en promedio 46.6 nacimientos por cada 1,000 mujeres de entre 15 y 19 años; presentando menores tasas de natalidad en adolescentes República de Corea (1.4), Suiza (2.8) y Singapur (3.5), mientras que, las mayores tasas se muestran en Níger (186.5), Mali (169.1) y Chad (161.1).

En la dimensión del empoderamiento, con relación a los escaños parlamentarios, la media de representación femenina para dicho año es del 24\%, presentando los mayores porcentajes de mujeres en los parlamentos Rwanda (55.7\%), Cuba (53.2\%) y Bolivia (51.8\%), mientras que los países con menor representación parlamentaria femenina son Yemen (1\%), Haití (2.7\%) Maldivas (4.6\%) y Kuwait (4.6\%). Se observa también que el promedio de mujeres de 25 años y mayores con al menos educación secundaria en los países analizados es del 61.59\%, teniendo los mayores porcentajes Canadá, Islandia, Finlandia, Austria, Luxemburgo, Estonia, Chequia y Letonia, con el 100\%, mientras que los países con los menores porcentajes son Chad (1.7\%), Niger (4.7\%) y Burkina Faso (6.1\%).

En la dimensión del mercado laboral, se observa que el promedio de participación de mujeres mayores de 15 años en los países analizados es del 51.85\%, teniendo la mayor participación en Ruanda (83.9\%), Nepal (82.8\%), Burundi (80.4\%), Mozambique (82.1\%) 
y Chad (80.2\%). Por otro lado, los países con menor participación femenina son Yemen (5.8\%), Iraq (11.6\%), Jordania (14.4\%) y República Árabe Siria (14.4\%), lo cual muestra que, en diversas sociedades, además de la educación y el crecimiento económico, la cultura y los roles de género inciden directamente en que las mujeres se desenvuelvan principalmente en el ámbito reproductivo y del cuidado del hogar.

\subsection{Matriz de correlaciones}

En la siguiente matriz de correlaciones generada por el modelo (ver tabla 2), se pueden identificar el grado en el que cada componente femenino del IDG es explicado por las otras variables femeninas del índice, mostrando un valor determinante de .019, lo cual indica que las variables del IDG se encuentran linealmente relacionadas, por lo que el análisis factorial es pertinente para analizarlas.

Tabla 2. Matriz de correlaciones.

\begin{tabular}{|c|c|c|c|c|c|c|c|}
\hline \multicolumn{8}{|c|}{ Matriz de correlaciones $^{a}$} \\
\hline & & $\begin{array}{c}\text { Índice } \\
\text { Desigualdad } \\
\text { de Género }\end{array}$ & \begin{tabular}{|c|} 
Tasa \\
Mortalidad \\
Materna \\
\end{tabular} & $\begin{array}{c}\text { Tasa } \\
\text { natalidad en } \\
\text { adolescentes }\end{array}$ & $\begin{array}{c}\text { Mujeres } \\
\text { en escaños } \\
\text { parlamentarios }\end{array}$ & $\begin{array}{l}\text { Mujeres con } \\
\text { secundaria }\end{array}$ & $\begin{array}{c}\text { Mujeres } \\
\text { en fuerza } \\
\text { laboral }\end{array}$ \\
\hline \multirow[t]{6}{*}{ Correlación } & $\begin{array}{l}\text { Índice Desigualdad } \\
\text { de Género }\end{array}$ & 1.000 & .724 & .808 & -.396 & -.806 & -.031 \\
\hline & $\begin{array}{l}\text { Tasa Mortalidad } \\
\text { Materna }\end{array}$ & .724 & 1.000 & .734 & -182 & -.718 & .262 \\
\hline & $\begin{array}{l}\text { Tasa natalidad en } \\
\text { adolescentes }\end{array}$ & .808 & .734 & 1.000 & -.114 & -.695 & .286 \\
\hline & $\begin{array}{l}\text { Mujeres en escaños } \\
\text { parlamentarios }\end{array}$ & -.396 & -.182 & -.114 & 1.000 & .150 & .287 \\
\hline & $\begin{array}{l}\text { Mujeres con } \\
\text { secundaria }\end{array}$ & -.806 & -.718 & -.695 & .150 & 1.000 & -.095 \\
\hline & $\begin{array}{l}\text { Mujeres en fuerza } \\
\text { laboral }\end{array}$ & -.031 & .262 & .286 & .287 & -.095 & 1.000 \\
\hline \multirow[t]{6}{*}{$\begin{array}{l}\text { Sig. } \\
\text { (unilateral) }\end{array}$} & $\begin{array}{l}\text { Índice Desigualdad } \\
\text { de Género }\end{array}$ & & .000 & .000 & .000 & .000 & .348 \\
\hline & $\begin{array}{l}\text { Tasa Mortalidad } \\
\text { Materna }\end{array}$ & .000 & & .000 & .010 & .000 & .000 \\
\hline & $\begin{array}{l}\text { Tasa natalidad en } \\
\text { adolescentes }\end{array}$ & .000 & .000 & & .075 & .000 & .000 \\
\hline & $\begin{array}{l}\text { Mujeres en escaños } \\
\text { parlamentarios }\end{array}$ & .000 & .010 & .075 & & .029 & .000 \\
\hline & $\begin{array}{l}\text { Mujeres con } \\
\text { secundaria }\end{array}$ & .000 & .000 & .000 & .029 & & .114 \\
\hline & $\begin{array}{l}\text { Mujeres en fuerza } \\
\text { laboral }\end{array}$ & .348 & .000 & .000 & .000 & .114 & \\
\hline
\end{tabular}

Fuente: Elaboración propia en SPSS, con datos del PNUD, 2021. 
En esta matriz se observa primeramente, que la muerte materna puede explicarse en gran medida por la tasa de natalidad en adolescentes, que presenta una relación positiva con un coeficiente de 0.734 y por la educación secundaria en las mujeres, que tiene una relación negativa con un valor de -0.718 , lo cual indicaría -en concordancia con la literatura citada-, que a mayor tasa de natalidad en adolescentes corresponde una mayor tasa de mortalidad materna, mientras que a mayor educación secundaria en las mujeres, corresponde una menor mortalidad materna. Así mismo, la tasa de natalidad en adolescentes puede explicarse en gran medida por la educación secundaria recibida por las mujeres, con un coeficiente negativo de -.695, indicando que a mayor educación secundaria femenina corresponde también una menor tasa de natalidad en adolescentes.

En cuanto a la ocupación de las mujeres en escaños parlamentarios y la participación femenina en la fuerza de trabajo, se observa que no son determinados por los demás componentes del IDG (muerte materna, natalidad en adolescentes y educación secundaria de las mujeres), ya que todos presentan coeficientes con valores débiles menores al 0.3.

\subsection{Prueba de KMO y Bartlett}

La siguiente tabla muestra los resultados de la prueba KMO y Bartlett, observándose en el modelo un valor KMO de 0.697 (ver tabla 3), lo cual indica que las correlaciones de los pares de variables pueden explicarse por otros factores latentes. Asimismo, la tabla contiene el resultado de la prueba de esfericidad de Bartlett, mostrando un valor de significación de 0.000 , lo que corrobora la correlación de las variables y la validez del modelo.

Tabla 3. Prueba de KMO y Bartlett.

\begin{tabular}{|l|l|r|}
\hline \multicolumn{2}{|c|}{ Prueba de KM0 y Bartlett } \\
\hline Medida Kaiser-Meyer-Olkin de adecuación de muestreo & .697 \\
\hline \multirow{3}{*}{ Prueba de esfericidad de Bartlett } & Aprox. Chi-cuadrado & 620.761 \\
\cline { 2 - 3 } & gl & 15 \\
\cline { 2 - 3 } & Sig. & .000 \\
\hline
\end{tabular}

Fuente: Elaboración propia en SPSS, con datos del PNUD, 2021.

\subsection{Extracción de componentes}

Como se puede apreciar en la tabla 4, adicionalmente a los factores contemplados en el IDG, existen dos principales factores subyacentes en el índice 2020, mostrando que el primer factor explica el 55.78 por ciento de la varianza total del IDG, mientras el segundo factor lo explica en un 22.89 por ciento. Ambos componentes presentan autovalores mayores a 1, explicando en conjunto el $78.69 \%$ de la varianza total del índice, por lo que son los componentes extraídos en el modelo. 
Tabla 4. Extracción de factores.

\begin{tabular}{|l|c|c|c|c|c|c|}
\hline \multicolumn{7}{|c|}{ Varianza total explicada } \\
\hline \multirow{2}{*}{ Componente } & Total & \% de varianza & \% acumulado & Total & \% de varianza & \% acumulado \\
\cline { 2 - 7 } & 3.347 & 55.788 & 55.788 & 3.347 & 55.788 & 55.788 \\
\hline 2 & 1.373 & 22.891 & 78.679 & 1.373 & 22.891 & 78.679 \\
\hline 3 & .641 & 10.690 & 89.369 & & & \\
\hline 4 & .294 & 4.899 & 94.268 & & & \\
\hline 5 & .258 & 4.295 & 98.563 & & & \\
\hline 6 & .086 & 1.437 & 100.000 & & & \\
\hline
\end{tabular}

Método de extracción: análisis de componentes principales.

Fuente: Elaboración propia en SPSS, con datos del PNUD, 2021.

Así mismo, como se puede apreciar en la gráfica 1 denominada de sedimentación o de caída, se reitera la principal importancia de dos factores subyacentes al IDG en 2020 en los países analizados, con una marcada importancia para el primer factor.

Gráfica 1. Gráfica de caída.

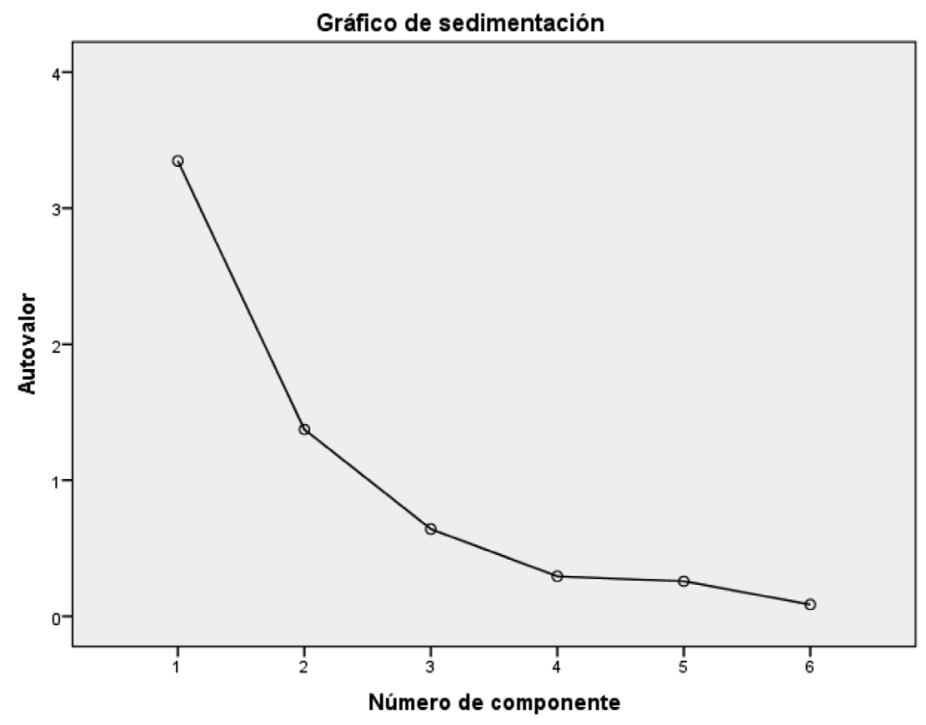

Fuente: Elaboración propia en SPSS, con datos del PNUD, 2021.

\subsection{Factores subyacentes en el IDG 2020}

El primer factor subyacente del IDG encontrado en el modelo corresponde al rol de género femenino, que encuadra a las mujeres como principales responsables del cuidado del hogar y de las personas, dado que, como ya se ha señalado, una causa estructural que limita alcanzar la igualdad de género, es la distribución inequitativa del poder entre hombres y mujeres desde el ámbito privado, basada en roles de género diferenciados y complementarios, que han situado culturalmente a las mujeres dentro del ámbito privado con un rol predominantemente reproductivo y de cuidado del hogar, haciéndolas principales 
responsables de la carga del trabajo doméstico y del cuidado de las personas, ya sean niños, enfermos o ancianos, lo cual, limita directamente su incorporación y desempeño en diferentes ámbitos de la esfera pública y las condiciona a la subordinación masculina, incluso del tipo físico y reproductivo.

Tabla 5. Extracción de factores.

\begin{tabular}{|l|c|c|}
\hline \multirow{2}{*}{} & \multicolumn{2}{|c|}{ Matriz de componente $^{\mathbf{a}}$} \\
\cline { 2 - 3 } & $\mathbf{1}$ & \multicolumn{1}{c|}{ Componente $^{-1}$} \\
\hline Índice Desigualdad de Género & .930 & -.227 \\
\hline Tasa natalidad en adolescentes & .895 & .179 \\
\hline Tasa Mortalidad Materna & .881 & .131 \\
\hline Mujeres con secundaria & -.881 & -.003 \\
\hline Mujeres en fuerza laboral & .194 & .833 \\
\hline Mujeres en escaños parlamentarios & -.301 & .761 \\
\hline
\end{tabular}

Método de extracción: análisis de componentes principales.

a. 2 componentes extraídos.

Fuente: Elaboración propia en SPSS, con datos del PNUD, 2021.

De esta manera, la matriz de componentes permite explicar que la posición predominante de las mujeres en el ámbito doméstico y reproductivo, impacta de forma importante en los cuatro primeros elementos de la matriz (ver tabla 5), ya que los valores que presenta son cercanos a la unidad, afectando de forma más importante al IDG (0.93), seguido de la tasa de natalidad en adolescentes (0.895), la mortalidad materna (0.881) y la proporción de mujeres con educación secundaria $(-0.881)$, siendo así que a mayor carga y responsabilidad femenina en el hogar, existe, mayor tasa de natalidad en adolescentes, mayor tasa de mortalidad materna y menos mujeres con al menos educación de nivel secundaria, que en conjunto se refleja en una mayor desigualdad de género.

Asimismo, se puede observar que dicha carga y responsabilidades en el hogar tiene poco peso en la incorporación de las mujeres a la fuerza laboral remunerada (.194), dado que, como se ha señalado, el que las mujeres participen en el mercado laboral, no las excluye de las responsabilidades del cuidado del hogar e incluso se observa que, a menor grado de desarrollo económico de los países, existe un mayor número de mujeres que buscan un trabajo remunerado. Se observa también que este aspecto afecta con signo negativo (-301) la proporción de escaños parlamentarios ocupados por mujeres, lo cual sugiere que, a mayor responsabilidad de las mujeres en el ámbito doméstico, corresponde una menor proporción de representación femenina parlamentaria.

En los resultados obtenidos se determina que el segundo factor subyacente en la desigualdad de género, con un coeficiente de -.227, es la educación y capacitación que reciben las mujeres en niveles superiores de instrucción, ya que, de acuerdo con Aguayo y Lamelas (2011), Angulo, et. al (2012) y CEPAL (2005), les representa el capital humano con el que las personas pueden incorporarse con mejores condiciones al ámbito público 
y al mercado laboral, por lo cual, a mayor educación profesional y capacitación de las mujeres, corresponde un mayor capital humano, con el cual se espera un mayor empoderamiento femenino.

Los resultados muestran que este factor tiene mayor influencia con una relación positiva en las variables de participación femenina en la fuerza de trabajo (0.833) y la ocupación de mujeres en escaños parlamentarios (0.761) y muestra una relación negativa con el IDG (-227), esperando entonces que, a mayor educación profesional y capacitación femenina, corresponda una menor desigualdad de género, con mayor participación en el mercado laboral y mayor representación parlamentaria, que como ya se señaló, ambos factores guardan poca relación con los logros en educación del nivel secundaria. Asimismo, se observa que este factor no muestra una relación significativa con las variables de natalidad en adolescentes, mortalidad materna, ni logros de las mujeres en educación secundaria, variables que tienen mayor relación con el primer factor subyacente.

\section{Conclusiones}

A pesar de los importantes logros obtenidos en la lucha por alcanzar la igualdad de género, a nivel global persisten importantes desigualdades en las condiciones en que viven mujeres y hombres, las cuales han generado amplias brechas de género en diferentes dimensiones. Diversos autores han resaltado el hecho de que mujeres y hombres hemos sido encuadrados en comportamientos socialmente aceptables correspondientes a un rol de género determinado, cuyo constructo se ha basado en las diferencias biológicas y la división sexual del trabajo, conteniendo una fuerte carga de dominación masculina, que ha propiciado el que la mujer quede culturalmente enmarcada dentro del ámbito reproductivo y del cuidado del hogar y las personas, por lo cual -independientemente de realizar o no algún trabajo remunerado- las mujeres siguen siendo las principales responsables de las labores domésticas y el cuidado de las personas dependientes, teniendo menor acceso a educación y capacitación acorde con las necesidades del mercado laboral, derivando en desventajas para ellas en los ámbitos público y privado, que afecta negativamente la expansión y disfrute de todas las libertades y oportunidades que el nivel de desarrollo pudiera brindarles.

El IDG es un indicador de referencia mundial que ha permitido dimensionar la pérdida de desarrollo humano por casusa de algunas desigualdades en las condiciones en que viven hombres y mujeres, permitiendo contar con mayores elementos de análisis de dicho problema público que afecta principalmente a las mujeres e incorporarlo a la agenda política con mayor fuerza. De esta forma, se han formulado diversas políticas públicas que toman como referencia el IDG en busca de mejorar las condiciones de las mujeres principalmente en las dimensiones que actualmente contempla, es decir, políticas para la prevención de la muerte materna y el embarazo adolescente, acceso de las mujeres a la educación básica y secundaria, mayor participación femenina dentro de los escaños parlamentarios, así como mayor participación de las mujeres en el mercado de trabajo, lo cual ha permitido ir generando avances importantes hacia la igualdad en dichas temáticas. 
Sin embargo, el IDG no contempla en su medición otros factores estructurales de la desigualdad de género, considerando dentro de la dimensión del empoderamiento solamente los logros en la educación secundaria que no necesariamente constituyen el capital humano necesario para lograr un pleno empoderamiento; asimismo, la dimensión del mercado laboral favorece el análisis de la participación en el trabajo remunerado, dejando de lado la participación en el trabajo no remunerado del hogar que aporta un gran valor social.

Como se ha comprobado con el modelo, dos importantes factores subyacentes de la desigualdad de género que capta el IDG son la inequitativa distribución de la carga de trabajo en el hogar y el insuficiente aprovechamiento del capital humano de las mujeres, factores que han sido menos cuantificados sistemáticamente por la mayoría de los países y menos abordados dentro de las agendas de gobierno. El modelo de análisis factorial mostró que cuanto mayor sea la responsabilidad de las mujeres en el ámbito doméstico, mayor será la desigualdad de género, la tasa de natalidad en adolescentes, la tasa de mortalidad materna, y menor será la proporción de mujeres con educación secundaria, mientras que cuanto mayor sea la proporción de mujeres con educación y capacitación en niveles superiores de instrucción, habrá menor desigualdad de género, con mayor proporción de mujeres en el mercado laboral y mayor representación parlamentaria femenina.

Se considera importante que estos factores puedan integrarse en las mediciones sistemáticas de la desigualdad de género, con indicadores sobre el tiempo que destinan las mujeres y hombres a realizar el trabajo doméstico y el cuidado de niños, enfermos y ancianos, así como los logros educativos en el nivel superior de ambos géneros, con lo cual, se pudiera tener un mayor alcance en la medición y evolución de la desigualdad de género, permitiendo también contar con mejores indicadores para realizar comparativas entre regiones y países.

Se propone en consecuencia, generar un IDG ampliado, que permita dimensionar otros factores estructurales de la desigualdad que fomentan la exclusión de las mujeres desde el ámbito privado y propician desventajas para ellas en otras dimensiones, de tal forma que las políticas públicas incluyan mayores elementos en su análisis, que puedan derivar en acciones afirmativas para propiciar un cambio cultural, que realmente apoye a que mujeres y hombres cuenten con las mismas condiciones y oportunidades desde el ámbito privado, coadyuvando a eliminar el aislamiento de las mujeres en la esfera reproductiva y del cuidado del hogar y de las personas, así como fomentar su incorporación a niveles más elevados de educación y capacitación, para conformar el suficiente capital humano con el cual puedan incorporarse adecuadamente en el mercado laboral y participar activamente en todos los niveles de la esfera pública con las mismas oportunidades y condiciones que los hombres, lo cual maximizaría el potencial humano de las mujeres, contribuyendo en mayor medida a los procesos de desarrollo con equidad de los países.

\section{Bibliografía}

AGUILAR MONTES DE OCA, YESSICA PAOLA, VALDEZ MEDINA, JOSÉ LUIS, GONZÁLESARRATIA LÓPEZ-FUENTES NORMA IVONNE y GONZÁLEZ ESCOBAR, SERGIO (2013). 
Los roles de género de los hombres y las mujeres en el México contemporáneo. Enseñanza e investigación en psicología, 18(2), 207-224. México.

AGUAYO, EVA y LAMELAS, NÉLIDA (2011). Educación y empleo: Desigualdad de género en las regiones mexicanas. 2000-2005. Estudios Feministas, Florianópolis, 19(3): 733-750.

ANGULO, GRACE, QUEJADA, RAÚL y YÁNEZ MARTHA (2012). “Educación, mercado de trabajo y satisfacción laboral: el problema de las teorías del capital humano y señalización de mercado". México. Revista de la educación superior. 41(163), 51-66.

CALLEALTA, FRANCISCO JAVIER. (2005). "New Measure of Dissimilarity Between Distributions: Aplication to the Analysys of Income Distributions Convergence in the European Union". Departamento de Estadísitca, Estructura Económica y O.E.I., Madrid, España, 42-52.

COMISIÓN ECONÓMICA PARA AMÉRICA LATINA (CEPAL, 2004). Caminos hacia la equidad de género en América Latina y el Caribe. México. 9a. Conferencia regional sobre la mujer en América latina y el Caribe. México: CEPAL. Recuperado en octubre de 2018. Versión electrónica disponible en: https://repositorio.cepal.org/bitstream/ handle/11362/16577/1/S2004062_es.pdf

COMISIÓN ECONÓMICA PARA AMÉRICA LATINA (CEPAL, 2005). Objetivos de Desarrollo del Milenio: una mirada desde América Latina y el Caribe. Santiago de Chile: CEPAL. COMISIÓN ECONÓMICA PARA AMÉRICA LATINA (CEPAL, 2018). Agenda 2030 y los Objetivos de Desarrollo Sostenible. Una oportunidad para América Latina y el Caribe. Santiago de Chile: CEPAL. 17-01256.

COX, TREVOR F. y COX, MICHAEL A. (1994). Multidimensional Scaling. Londres: Chapman y Hall.

DAEREN, LIEVE (2001). "Enfoque de género en la política económica-laboral. El estado del arte en América Latina y el Caribe". Mujer y Desarrollo. CEPAL. Disponible en https://repositorio.cepal.org/bitstream/handle/11362/5874/S01020192_es.pdf? sequence=1\&isAllowed $=y$

DIARIO OFICIAL DE LA FEDERACIÓN (DOF) (2013). Programa Nacional para la Igualdad de Oportunidades y no Discriminación contra las Mujeres (PROIGUALDAD) 20132018. México: Cámara de Diputados del H. Congreso de la Unión.

FONDO DE POBLACIÓN DE LAS NACIONES UNIDAS (UNFPA) (2020). Consecuencias socioeconómicas del embarazo en adolescentes en México. México: UNFPA.

GAMBOA MONTEJANO, CLAUDIA y VALDÉS ROBLEDO, SANDRA (2015). Mortalidad materna en México. Marco conceptual, derecho comparado, políticas públicas, tratados internacionales, estadísticas y opiniones especializadas. México: Dirección General de Servicios de Documentación, Información y Análisis.

GUILLERMO PEÓN, SYLVIA BEATRIZ y GARCÍA PÉREZ, ISRAEL GERARDO (2010), Índice de Competitividad para el Municipio de Puebla. Universidad Autónoma de Puebla, Puebla, México.

IKANOWICZ, OCHMAN y BARBARA MARTA (2016). Políticas sociales y empoderamiento de las mujeres. Una promesa incumplida. Estudios Políticos, 48, Instituto de Estudios 
Políticos, Universidad de Antioquia, pp. 32- 51. DOI: 10.17533/udea.espo.n48a03 LAMAS, MARTHA (1986). "La antropología feminista y la categoría" género"'. Nueva antropología, 8(30), 173-198.

LÓPEZ-ROLDÁN, PEDRO y FACHELLI, SANDRA (2016). Metodología de la Investigación Social Cuantitativa. Bellaterra (Cerdanyola del Vallès): Dipòsit Digital de Documents, Universitat Autònoma de Barcelona. 1aㅡ edición, versión 3. Edición digital:

MAGAÑA GARCÍA, PAULINA (2018). La perspectiva de género en las políticas públicas como medio para lograr el desarrollo humano en Michoacán. Tesis para obtener el grado de Maestra en Derecho Administrativo. México: Universidad Michoacana de San Nicolás de Hidalgo.

MARTÍNEZ CANALES, DELIA (2015). El techo de cristal para mujeres funcionarias en el IPN. Tesis para obtener el grado de Maestro en Administración de Negocios. México: Instituto Politécnico Nacional.

MERINO, MAURICIO (2013). Políticas públicas: Ensayo sobre la intervención del Estado en la solución de problemas públicos. México: CIDE.

NOGUERA, JOSÉ ANTONIO (2002). El concepto de trabajo y la teoría social crítica. Papers: Revista de Sociología, 141-168. Barcelona.

ORGANIZACIÓN DE LAS NACIONES UNIDAS (2014). Empoderamiento político de las mujeres: marco para una acción estratégica. América Latina y el Caribe (2014-2017). ONU Mujeres. Recuperado en marzo 2019. Versión electrónica disponible en: http:// www.observatoriomujeresnl.mx/docs/empoderamiento_politico_de_las_mujeresnew2-cn.pdf

ORGANIZACIÓN DE NACIONES UNIDAS (2015). Transformar a nuestro mundo: la Agenda 2030 para el Desarrollo Sostenible. Suiza: ONU.

ONU Mujeres (2015). Los beneficios del empoderamiento económico. Noticias, Organización de las Naciones Unidas. Disponible en: https://www.unwomen.org/es/whatwe-do/economic-empowerment/facts-and-figures.

PATIL, SHREENATH y BONASODE, CHANDRASHEKAR (2018). Recent trends in women empowerment. Recuperado en octubre 2018. Versión electrónica disponible en: http:// www.academia.edu/11259518/RECENT_TRENDS_IN_WOMEN_EMPOWERMENT

PROGRAMA DE LAS NACIONES UNIDAS PARA EL DESARROLLO (PNUD) (2010). Informe sobre Desarrollo Humano, 2010. La verdadera riqueza de las naciones. Edición del vigésimo aniversario. Estados Unidos: PNUD

PROGRAMA DE LAS NACIONES UNIDAS PARA EL DESARROLLO (PNUD) (2014). Indicadores de Desarrollo Humano y Género en México: nueva metodología. Identificar las barreras para lograr la igualdad. México: PNUD

PROGRAMA DE LAS NACIONES UNIDAS PARA EL DESARROLLO (PNUD) (2019). Informe sobre Desarrollo Humano 2019. Más allá del ingreso, más allá de los promedios, más allá del presente: Desigualdades del desarrollo humano en el siglo XXI. Estados Unidos: PNUD

PROGRAMA DE LAS NACIONES UNIDAS PARA EL DESARROLLO (PNUD) (2021). La próxima frontera. El desarrollo humano y el Antropoceno. Estados Unidos: PNUD. 
RAMOS PADILLA, MIGUEL (2006). La salud sexual y la salud reproductiva desde la perspectiva de género. Revista peruana de medicina experimental y salud pública, 23(3), 201-220.

SEN, AMARTYA (2000). Desarrollo y libertad. Buenos Aires: Editorial Planeta.

ZAMORA-TORRES, AMÉRICA I. y THALHEIM, LUISE (2020). El Modelo Mexicano de Formación Dual como modelo educativo en pro de la inserción laboral de los jóvenes en México. Revista iberoamericana de educación superior, 11(31), 48-67.

ZAMUDIO SÁNCHEZ, FRANCISCO JOSÉ, AYALA CARRILLO, MARÍA DEL ROSARIO y ARANA OVALLE, ROCÍO IVETTE (2014). Mujeres y hombres: Desigualdades de género en el contexto mexicano. Estudios sociales (Hermosillo, Son.), 22(44), 251-279. México.

Tabla 1: muestra como principales estadísticos descriptivos, la media y la desviación estándar de los 161 países analizados, para cada una de las 5 variables que integra el modelo.

Tabla 2: en esta tabla generada por el modelo, se pueden identificar el grado en el que cada componente femenino del IDG es explicado por otras variables, teniendo una mayor fuerza al acercarse su valor a la unidad, ya sea con una relación positiva o negativa.

Tabla 3: muestra los resultados de la prueba KMO y Bartlett, con un valor de significación de 0.0, mostrando que las variables analizadas se encuentran correlacionadas y pueden explicarse por otras variables.

Tabla 4: al realizar la extracción de factores, se muestra la existencia de dos principales factores subyacentes en las variables analizadas del IDG, explicando el primer factor el 55.78\% de la varianza total del modelo, mientras el segundo factor lo explica en un $22.89 \%$.

Tabla 5: la matriz de componentes muestra el peso de la relación (positiva o negativa) que tienen los dos factores subyacentes identificados con cada una de las variables analizadas en el modelo.

Gráfica 1: la gráfica de caída o sedimentación confirma la existencia de dos factores subyacentes a las variables analizadas en el modelo. 